\title{
RESVERATROL EXERTS AN ANTIPROLIFERATIVE EFFECT IN PANCREATIC CANCER CELLS BY INDUCING MITOCHONDRIAL-MEDIATED APOPTOSIS
}

\author{
YONGYUN LUO'”, DI LIU'", XINQING WANG', XIAOPING YE², NAN LI', \\ YANG ZHANG', JIANJUN SONG ${ }^{1}$ and QI WANG'*
}

'Department of Hepatobiliary Surgery,

${ }^{2}$ Department of Colorectal Surgery, General Hospital of Ningxia Medical University, Yinchuan, Ningxia, China, 750004

\begin{abstract}
Resveratrol, an active natural phytoalexin compound mainly derived from grapes exhibits numerous pharmacological activities including anticancer activity. The current evaluate the anticancer potential of resveratrol against pancreatic cancer cells. Our results demonstrate that resveratrol exhibits significant antiproliferative activity against a panel of pancreatic cancer cell lines. Quantification of apoptotic cells after staining with annexin $\mathrm{V}$ and propidium iodide (PI) by flow cytometry revealed a significant number of tumor cells undergo apoptosis at higher doses of resveratrol. Further, confirmation by immunoblotting reveals a significant increase in the cleaved product of PARP1 and caspase-3 with a notable expression of mitochondrial resident protein cytochrome-3 at higher doses of resveratrol. Additionally, we observe a high production of reactive oxygen species (ROS) by conversion of DCFDH2A into green fluorescein color when pancreatic cancer cells were exposed to the dose-dependent concentration of resveratrol. To confirm the mitochondrial-dependent apoptosis, JC-1 staining of pancreatic cells exposed to varying doses of resveratrol reveals a significant population of pancreatic cancer cells was stained with JC-1 dye at higher doses of resveratrol indicating the depolarization of mitochondrial membrane potential was induced in pancreatic cancer cells upon resveratrol treatment to kill cells by mitochondrial-dependent apoptosis. In summary, resveratrol exhibits an antiproliferative effect in pancreatic cells by simultaneously induces ROS production and mitochondrial-dependent apoptosis.
\end{abstract}

Keywords: resveratrol, pancreatic cancer, apoptosis, mitochondria, flow cytometry

Pancreatic cancer is one of the deadliest cancers and the fourth leading cause of mortality among all the cancers in the USA. It is estimated that pancreatic cancer will be the second in terms of mortality in the USA by 2030 (1). The major reasons are poor prognosis and high mortality. The low survival rate $(6 \%)$ of pancreatic cancer patients is due to latestage diagnosis and asymptomatic even at an advanced stage $(2,3)$. Approximately $20 \%$ of pancreatic patients are eligible for initial resection and most patients among them with complete resection have significant chances of recurrence with a fiveyear survival period not more than $25 \%$ (4). The major risk factors which increase the incidence of pancreatic cancer are familial genetic risk towards pancreatic cancer, cigarette smoking, diabetes, chronic pancreatitis, and no screening program for these high-risk patients (5).
At the molecular level, pancreatic cancer patients develop mutations in four important genes that regulate various signaling pathways (6). These four genes are KRAS, CDKN2A, TP53, and SMAD4. $K R A S$ a protooncogene that encodes small GTPase and facilitates signaling to growth factor receptors (7). However, CDKN2A, TP53, and SMAD4 are tumor suppressor genes control cell cycle, cellular stress, and transforming growth factor- $\beta$ (TGF- $\beta$ ) signaling respectively $(8,9)$.

The therapeutic options are limited for pancreatic cancer patients (10). Most of the patients are not resectable and have a maximum tendency of metastatic or have developed metastasis (11). For those patient's chemotherapy and radiotherapy is the only option that too is ineffective with adverse side effects (12). Therefore, there is a need for novel agents that not only kills tumor cells effectively but

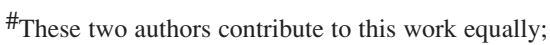

* Corresponding author: e-mail: QiWang69@yahoo.com
} 
also have the least side effects on normal cells. The natural compounds obtained from natural sources (plant sources) have complex structures and have great potential to target numerous cancer signaling proteins to control the uncontrolled proliferation of tumor cells by inducing cell killing (apoptosis, autophagy) with the least effect on normal cells (13, 14).

Resveratrol is a natural phytoalexin, a small molecular weight compound, and mainly derived from grapes (15). Owing to serve as a polyphenolic antibiotic, resveratrol is used against many plantassociated pathogenic microorganisms including fungi and bacteria (16). Resveratrol has been studied extensively for the last three decades for its pharmacological activities (17). Resveratrol exhibits numerous pharmacological activities such as antiplatelet aggregation, anti-inflammatory, anti-atherogenic, antioxidant, immune-modulation, and antiproliferative activities (18). Despite having numerous activities, recent studies revealed that resveratrol has been used widely as a chemopreventive natural compound (19). The compound has been used to evaluate the anti-tumor effect against a wide variety of human cancers (20). Resveratrol mainly kills cancer cells by apoptosis (21). The apoptosis mechanism induced by resveratrol is operated through various signaling pathways such as CD95 (22), and TRAIL-mediated signaling to activate downstream proapoptosis proteins such as caspase-3, caspase-9, Bim. Bad and Bax (23).

Previous reports suggest that resveratrol induces an antiproliferative effect against pancreatic cancer cells. However, to the best of our knowledge, resveratrol against human pancreatic cancer cells has not been reported to exhibit an antiproliferative effect and induces mitochondrial-dependent apoptosis to kill pancreatic cancer cells. The current study evaluates the antiproliferative effect and cell death mechanism (mitochondrial-mediated apoptosis) induced by resveratrol in pancreatic cancer cells.

\section{MATERIALS AND METHODS}

\section{Chemicals, reagents, and antibodies}

Resveratrol, propidium iodide, dimethyl sulfoxide (DMSO), 2',7'-dichlorodihydrofluorescein diacetate (DCFDA), 5,5,6,6'-tetrachloro-1,1',3,3'tetraethylbenzimi-dazoylcarbocyanine iodide (JC-1) and annexin $\mathrm{V}$ apoptosis detection kit were purchased from Sigma Aldrich. The primary antibodies PARP1, Caspase-3, Bcl-2, Bax, and Cytochrome-3 were purchased from cell signaling technology. The Secondary antibodies IgG-tagged horseradish per- oxidase anti-mouse and anti-rabbit were procured from Santacruz Biotechnology.

\section{Cell lines and cell culture}

Pancreatic cancer cell lines PANC-1, Mia$\mathrm{PaCa}$, and Capan-2 were procured from the American Type Culture Collection (ATCC). All three cell lines were regularly maintained in culture media (RPMI supplemented with $10 \%$ FBS) in $5 \%$ $\mathrm{CO}_{2}$ incubator at $37^{\circ} \mathrm{C}$.

\section{Cell viability assay}

Cell proliferation of pancreatic cancer cells (PANC-1, Mia-PaCa, and Capan-2) upon treatment with varying concentrations of resveratrol was determined by MTT assay. Briefly, $5 \times 10^{3}$ pancreatic cancer cells were plated in each well of 96-well plates and let them adhere to the plate bottom surface. The next morning plated cells were exposed to varying concentrations of resveratrol along with control DMSO for $24 \mathrm{~h}$ and incubated in $5 \% \mathrm{CO}_{2}$ incubator at $37^{\circ} \mathrm{C}$. After the completion of time saturate each well with $20 \mu \mathrm{L}$ of $2.5 \mathrm{mg} / \mathrm{mL}$ MTT dye for the next 3-4 $\mathrm{h}$ and incubate in $5 \% \mathrm{CO}_{2}$ incubator at $37^{\circ} \mathrm{C}$. Add $150 \mu \mathrm{L}$ of DMSO in each well to dissolve formazan crystals formed during the incubation with MTT. Gently mix with vortex and read the plate at $570 \mathrm{~nm}$ and record the absorbance. The percentage of cell viability was calculated by the using latest GraphPad Prism.

\section{Annexin V staining for apoptosis detection using flow cytometry}

The quantification of apoptosis was performed by using flow cytometry. Briefly, $5 \times 10^{5}$ pancreatic cancer cells (Mia-PaCa) were seeded in each well of the 6-well plate and let adhere overnight. Next, morning cells were exposed to a dose-dependent concentration of resveratrol for $24 \mathrm{~h}$, washed with ice-cold PBS, harvested, resuspended and stain in binding buffer containing annexin V and PI for 15 $\min$ at $4^{\circ} \mathrm{C}$. After the completion of incubation stained cells were analyzed by flow cytometry to quantify the percentage of apoptotic cells.

\section{Detection of reactive oxygen species (ROS)}

Detection and estimation of ROS were analyzed by changing the colorless DCFHDA dye into a green-colored compound dichlorofluorescein (DCF). Briefly, $1 \times 10^{5}$ pancreatic cancer cells (Mia$\mathrm{PaCa}$ ) were seeded in each well of the 6-well plate and let adhere overnight. Next morning cells were exposed with varying concentrations of resveratrol in the presence and absence of $\mathrm{N}$-acetyl cysteine 
(NAC) along with ROS inducer $\mathrm{H}_{2} \mathrm{O}_{2}$ as positive control and a vehicle DMSO for $24 \mathrm{~h}$ incubated in $5 \% \mathrm{CO}_{2}$ incubator at $37^{\circ} \mathrm{C}$. After $24 \mathrm{~h}$, exposed cells were washed with PBS and stained with DCFHDA dye for the next 30 minutes in $5 \% \mathrm{CO}_{2}$ incubator at $37^{\circ} \mathrm{C}$. After the completion of incubation, cells were analyzed under a microplate reader and record the absorbance at an excitation wavelength of $488 \mathrm{~nm}$ and emission wavelength $525 \mathrm{~nm}$.

\section{Subcellular fractionation and immunoblotting}

Isolation of cytosolic and mitochondrial fractionation was performed to evaluate the role of mitochondrial-dependent apoptosis. Briefly, $5 \times 10^{5}$ pancreatic cancer cells (Mia-PaCa) were plated in each well of the 6-well plate and adhere to the bottom surface of the well overnight. Next, morning cells were exposed to varying concentration of resveratrol along with control DMSO for $24 \mathrm{~h}$ after the completion of time point, treated cells were gently washed with ice-cold PBS and resuspended and incubated in $500 \mu \mathrm{L}$ extraction lysis buffer (HEPES-KCl $20 \mathrm{mM}$ [pH 7.4], sucrose - $250 \mathrm{mM}$, PMSF - $10 \mathrm{mM}, \mathrm{KCl}$ - $10 \mathrm{mM}$, DTT - $1 \mathrm{mM}, \mathrm{MgCl}_{2}-1.5 \mathrm{mM}$, leupeptin $-10 \mu \mathrm{M}$, EGTA $-1 \mathrm{mM}$, and aprotinin $-10 \mu \mathrm{M}$ ) for $10 \mathrm{~min}$ on ice. Homogenization of cells was performed by passing 10 times through needle have diameter 26-gauge and homogenate thus obtained were centrifuged for $5 \mathrm{~min}$ at $1000 \mathrm{~g}$ to extract dam- age to fewer cells and nuclei. The supernatant thus obtained was recentrifuged at $4^{\circ} \mathrm{C}$ for $30 \mathrm{~min}$ at $12000 \mathrm{~g}$. The supernatant fraction now obtained is a cytosolic fraction, however, the pellet is mitochondria enriched fraction were again incubated on ice with mitochondrial lysis buffer in 1x PBS (NP-40 $1 \%$, SDS $-0.1 \%$, leupeptin $-1 \mu \mathrm{g} / \mathrm{mL}$, sodium deoxycholate $-0.5 \%$, aprotinin $-1 \mu \mathrm{g} / \mathrm{mL}$, PMSF $10 \mu \mathrm{g} / \mathrm{mL}$, DTT $-1 \mathrm{mM}$, and sodium orthovanadate $-2 \mathrm{mM}$ ) for next $20 \mathrm{~min}$. The lysis buffer was then centrifuged at $4^{\circ} \mathrm{C}$ for $5 \mathrm{~min}$ at $15000 \mathrm{~g}$. The supernatant thus obtained is enriched with mitochondrial content and some amount was used for protein determination by the standard Bradford method. The protein concentration was calculated, and an equal amount of $20 \mu \mathrm{g}$ of protein was loaded in each well SDS-PAGE, resolved, transferred on the PVDF membrane, and finally detected by using ECL analysis.

\section{Mitochondrial membrane potential}

The measurement of depolarization of mitochondrial membrane potential was used to authorize the role of the mitochondrial-mediated apoptotic pathway. Briefly, $5 \times 10^{5}$ pancreatic cancer (Mia$\mathrm{PaCa}$ ) were seeded in each well of 6-well and allowed to adhere to the bottom surface of the well overnight. The next morning, cells were exposed to varying concentrations of resveratrol along with
A

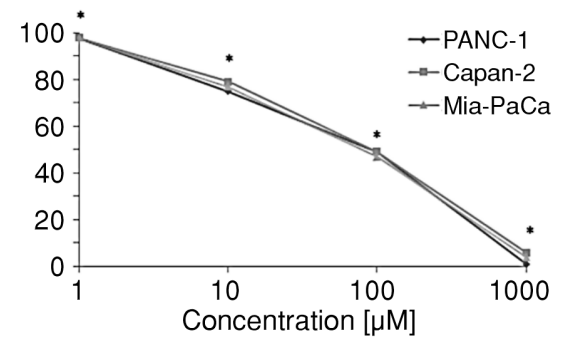

B

\begin{tabular}{c|c}
\hline Cell line & $\mathrm{IC}_{50}$ Value \\
\hline PANC-1 & $88.67 \pm 4.21$ \\
\hline Capan-2 & $90.34 \pm 4.89$ \\
\hline Mia-PaCa & $78.90 \pm 3.89$ \\
\hline
\end{tabular}

C
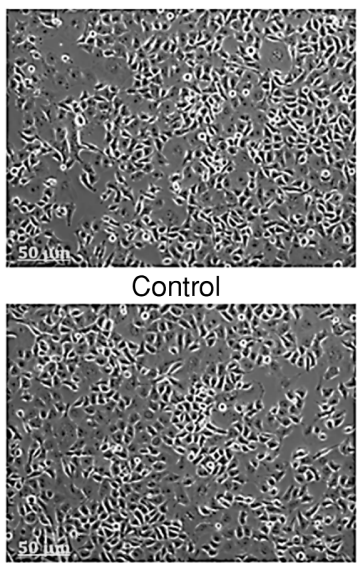

Resveratrol $100 \mu \mathrm{M}$

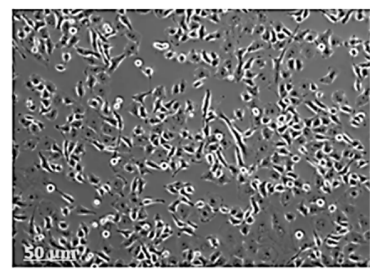

Resveratrol $50 \mu \mathrm{M}$

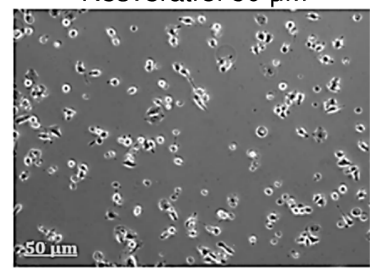

Resveratrol $150 \mu \mathrm{M}$

Figure 1. Resveratrol attenuates cell proliferation of pancreatic cancer cells. (A) The effect of resveratrol on the cell viability of pancreatic cancer cell lines (PANC-1, Capan-2, and Mia-PaCa) was determined by the MTT assay. (B) The $\mathrm{IC}_{50}$ value of pancreatic cancer cells (PANC-1, Capan-2, and Mia-PaCa) in a tabulated form after exposed to resveratrol for $24 \mathrm{~h}$. (C) Pancreatic cancer cells (Mia-PaCa) were treated with resveratrol in a concentration-dependent manner along with untreated control for $24 \mathrm{~h}$, to determine the morphological changes. Scale bar: $50 \mu \mathrm{m} ; 10 \mathrm{x}$. The data represent the mean value $\pm \mathrm{SE}$ of three independent experiments $* \mathrm{p}<0.05$. 
control DMSO for $24 \mathrm{~h}$. After the completion of the time point, cells were gently washed with PBS, followed by incubation, and staining with JC-1 dye for 15-20 $\mathrm{min}$ at room temperature. The JC-1 stained cells were analyzed using flow cytometry.

\section{Phase-contrast microscopy}

Briefly, $5 \times 10^{4}$ pancreatic cancer cells (Mia$\mathrm{PaCa}$ ) were seeded on the sterile coverslips inside the wells of the 6-well plate and allowed to adhere to the coverslips overnight. Next, morning wells containing coverslips adhered to cells were exposed to varying concentrations of resveratrol along with control DMSO for $24 \mathrm{~h}$. After the completion of the time point, cells were analyzed under a phase-contrast microscope to visualize any change in the morphology of cells after treatment with resveratrol.

\section{Statistical analysis:}

Data obtained from all the above experiments were presented as the mean \pm standard error of equal or more than three independent experiments. All the data were analyzed by one-way ANOVA. A twosided value of $* \mathrm{p}=0.05$ was statistically significant in all cases.

\section{RESULTS}

Resveratrol induces antiproliferative activity against pancreatic cancer cell lines

Previous studies revealed that resveratrol induces promising cytotoxic activity against a wide variety of human cancer cell lines. Therefore, we also intended to evaluate the antiproliferative effect of resveratrol against a panel of pancreatic cancer cells. (PANC-1, Mia-PaCa, and Capan-2). The effect of resveratrol on pancreatic cancer cells was determined by MTT assay. Our MTT assay analysis revealed that resveratrol initiates cell cytotoxicity effect against a panel of pancreatic cancer cells (PANC-1, Mia-PaCa, and Capan-2) at a lower dose of resveratrol $(10 \mu \mathrm{M})$. After taking absorbance readings from all the treated wells of 96-well plate exposed to varying concentrations of resveratrol for $(1 \mu \mathrm{M}$ to $1000 \mu \mathrm{M})$ for $24 \mathrm{~h}$, the data analysis was performed using GraphPad Prism software, we observed that the IC50 value of resveratrol is $88.67 \mu \mathrm{M}, 78.9 \mu \mathrm{M}$, and $90.34 \mu \mathrm{M}$ for PANC-1, Mia-PaCa, and Capan-2 cell lines, respectively (Fig. 1A). These data indicate that resveratrol showed a significant antiproliferative effect against a panel of pancreatic cell lines and the most promising effect was against Mia-PaCa cells.

Next, we sought to evaluate any morphological variations in the cellular morphology of cells after exposure with varying concentrations $(50,100$, and $150 \mu \mathrm{M})$ of resveratrol. Our phase-contrast microscopy results showed that a significant number of Mia-PaCa cells were killed and were observed as spherical cells floating on the culture medium when exposed at higher doses (100, and $150 \mu \mathrm{M})$ of resveratrol compared to control DMSO, (Fig. 1B), indicates that resveratrol is a prospective cytotoxic natural compound against pancreatic cancer cells.

\section{Resveratrol kills pancreatic cells by induction of apoptosis}

In the recent past, studies suggest that resveratrol induces cell killing by various modes in human cancer cells. Therefore, we intended to investigate the mode of cell death in pancreatic cancer cells (Mia-PaCa) upon treatment with varying doses of resveratrol $(50,100$, and $150 \mu \mathrm{M})$ along with staurosporine $(25 \mathrm{nM})$ as positive control and control DMSO as the vehicle. After treatment with varying doses of resveratrol for $24 \mathrm{~h}$, Mia-PaCa cells were harvested and stained with annexin V and PI and acquired the samples in flow cytometry. Our results revealed that resveratrol induces significant pancreatic cancer cell population towards apoptosis $35.3 \%$, $23.7 \%$, and $11.5 \%$ at doses $150 \mu \mathrm{M}, 100 \mu \mathrm{M}$, and 50 $\mu \mathrm{M}$, respectively when compared to $39.3 \%$ of positive control staurosporine and $3.6 \%$ of control DMSO (Figs. 2A and 2B). To further confirm the mechanism of resveratrol-mediated apoptosis we perform immunoblotting analysis. After exposing the pancreatic cells (Mia-PaCa) with varying doses of resveratrol $(50,100$, and $150 \mu \mathrm{M})$ for $24 \mathrm{~h}$, the lysate obtained from harvested cells were subjected to immunoblotting to check the protein expression of proapoptotic proteins. Our results demonstrated that there is a significant activation of caspase- 3 and PARP1 proapoptotic proteins at higher doses of resveratrol which was evidenced by cleaved fragment detected on the immunoblot of both caspase- 3 and PARP1 activated proteins. (Fig. 2C). These results indicate that resveratrol kills pancreatic cancer cells (Mia-PaCa) by apoptotic mode of cell death in a dose-dependent manner.

\section{Resveratrol promotes ROS generation in pancre- atic cancer cells:}

Numerous studies reveal that natural products kill human cancer cells by generating ROS production in vitro cellular models. We also intended to investigate whether pancreatic cancer cells (Mia$\mathrm{PaCa}$ ) induces ROS production upon treatment with resveratrol in the presence and absence of NAC (5 $\mathrm{mM}$ ), an antioxidant that inhibits ROS production. 
After treatment with resveratrol, (50, 100, and 150 $\mu \mathrm{M})$, Mia-PaCa cells were stained with DCFDA staining to detect any ROS production. The reaction was catalyzed by these free radicals which convert permeable colorless DCFDA into a green fluorescent product DCF (dichlorofluorescein). Interestingly, our results revealed that resveratrol induces significant ROS production in Mia-PaCa cells in a dosedependent manner. However, Mia-PaCa cells exposed to a higher dose of resveratrol $(150 \mu \mathrm{M})$ and NAC ( $5 \mathrm{mM}$ ) inhibits ROS production when compared with untreated control (Figs. 3A and 3B). These results indicate that induction of ROS by resveratrol might have augmented the apoptotic population of pancreatic cancer cells, thus plays a crucial role in cell death of pancreatic cancer cells.

\section{Resveratrol promotes mitochondrial-mediated apoptosis in pancreatic cancer cells.}

One of the hallmarks of mitochondrial-mediated cell death is the induction of ROS production. To investigate whether resveratrol activates mitochondrial-mediated apoptosis in pancreatic cells, we expose Mia-PaCa cells to varying concentrations of resveratrol for $24 \mathrm{~h}(50,100$, and $150 \mu \mathrm{M})$ for $24 \mathrm{~h}$.
After completion of treatment, cells were harvested and stained with JC-1 dye and acquired in flow cytometry to determine the changes in the depolarization of mitochondrial membrane potential. Our flow cytometry results suggest that a significant population of pancreatic cancer cells lose the mitochondrial membrane potential thereby undergoes mitochondrial-mediated apoptosis. at higher doses (Figs. 4A and 4B). Another striking feature of mitochondrial-mediated apoptosis is the secretion of mitochondrial resident protein cytochrome C. Our immunoblotting results of subcellular fraction lysates reveals that higher doses of resveratrol augment the secretion of cytochrome $\mathrm{C}$ in cytosolic fraction with sharp decrease and increase in expression of anti-apoptotic protein $\mathrm{Bcl}-2$ and proapoptotic protein Bax, respectively. (Fig. 4C). Collectively, these results suggest that resveratrol-mediates ROS production, which in turn augments mitochondrialdependent apoptosis in pancreatic cancer.

\section{DISCUSSION}

Pancreatic cancer is one of the deadliest cancers among all the cancers across the globe (24).
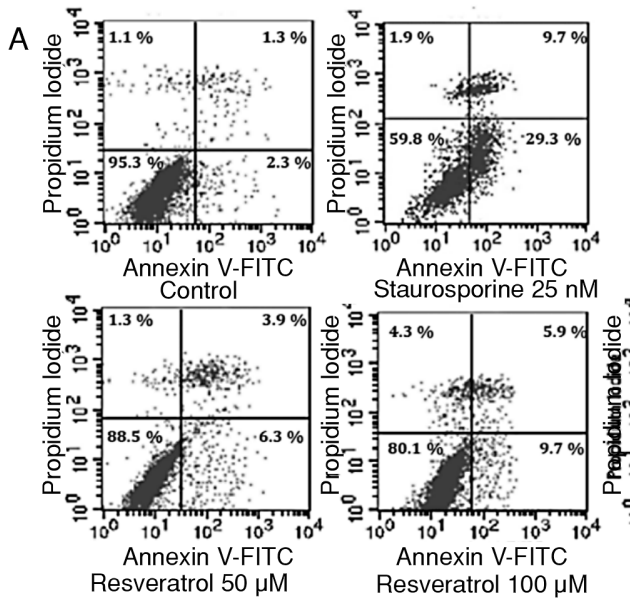

B

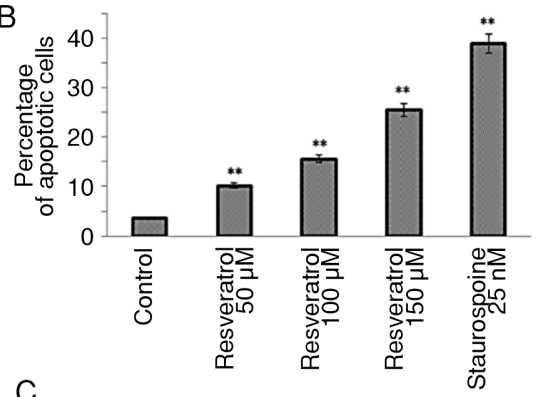

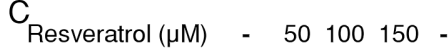
Staurosporine $(\mathrm{nM}) \quad-\quad-\quad-\quad-\quad 25$

Procaspase-3 $m-35 \mathrm{KDa}$

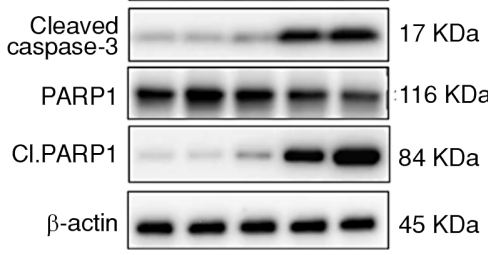

Figure 2. Resveratrol induces apoptosis in pancreatic cancer cells. A. Pancreatic cancer cells (Mia-PaCa) were exposed to indicated concentrations of resveratrol along with untreated control and positive control staurosporine $25 \mathrm{nM}$ for $24 \mathrm{~h}$ and then subjected to Annexin $\mathrm{V}$ FITC and propidium iodide staining and analyzed by FACS. (B) Bar diagram showing quantification of Annexin V FITC positive cells analyzed by FACS. (C) Immunoblotting analysis showing prominent PARP1 and caspase-3 cleavage in lanes where cells were exposed to higher concentrations of resveratrol and staurosporine $25 \mathrm{nM}$ treatment and internal control $\beta$-actin. The data represent the mean value $\pm \mathrm{SE}$ of three independent experiments $* * \mathrm{p}<0.01$. 
Owing to poor diagnosis, the mortality rate is alarmingly increasing in pancreatic cancer patients, and is believed that pancreatic cancer will be the second in rank by 2030 in the USA (25). Recent advancements in molecular therapeutics and diagnostics improved the survival rate of cancer patients significantly, however, in pancreatic cancer patients the survival rate statistics across the world have not improved substantially from last for decades (26). Owing to harboring some key mutations in genes KRAS, $C D K N 2 A, T P 53$, and SMAD4 which regulating crucial signaling pathways in pancreatic tumorigenesis, the current therapeutic modalities are ineffective in treating pancreatic cancer patients (27). Most of the patients are not resectable and have a maximum tendency of metastatic or have developed metastasis. For those patient's chemotherapy and radiotherapy is the only option that too is ineffective with adverse side effects (28). Therefore, there is a need for novel agents that not only kills tumor cells effectively but also have the least side effects on normal cells. The natural compound obtained from natural sources (plant sources) have a complex structure and has great potential to target various cancer signaling proteins to control the uncontrolled proliferation of tumor cells by inducing cell killing (apoptosis, autophagy) with the least effect on normal cells.

Resveratrol is a natural compound with promising anticancer activity against in vitro and in vivo mod- els of various cancers with low toxicity profile against normal cells (29). Resveratrol has a broad spectrum of intracellular molecular targets which plays a crucial role in the transformation and cellular growth during the development of tumorigeneses such as protein kinases, receptor tyrosine kinases, and cyclooxygenases (30). Resveratrol induces both autophagy and apoptosis mode of cell death in cancer cells, but it mainly kills cancer cells by apoptosis (31). The apoptosis mechanism induced by resveratrol is operated through various signaling pathways such as CD95, and TRAIL-mediated signaling to activate downstream proapoptotic proteins such as caspase-3, caspase-9, Bim Bad, and Bax (22, 23). Additionally, resveratrol has been demonstrated to arrest cell cycle at various stages of the cell cycle to attenuate cancer cell growth (32). Although reports suggest that resveratrol induces an antiproliferative effect against pancreatic cancer cells. However, to the best of our knowledge, resveratrol against human pancreatic cancer cells has not been reported to exhibit an antiproliferative effect and induces mitochondrial-dependent apoptosis to kill pancreatic cancer cells. The current study evaluates the antiproliferative effect and cell death mechanism (mitochondrial-mediated apoptosis) induced by resveratrol in pancreatic cancer cells.

The current study demonstrates the antiproliferative potential of resveratrol against a panel of

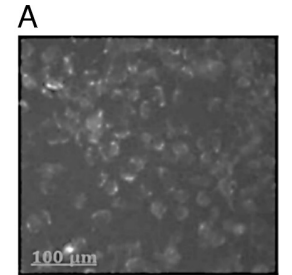

Control

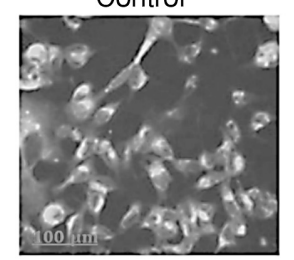

Resveratrol $100 \mu \mathrm{M}$

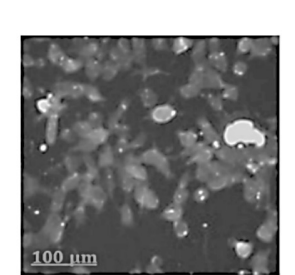

Resveratrol $50 \mu \mathrm{M}$

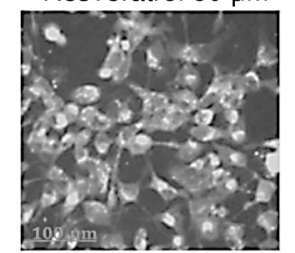

Resveratrol $150 \mu \mathrm{M}$

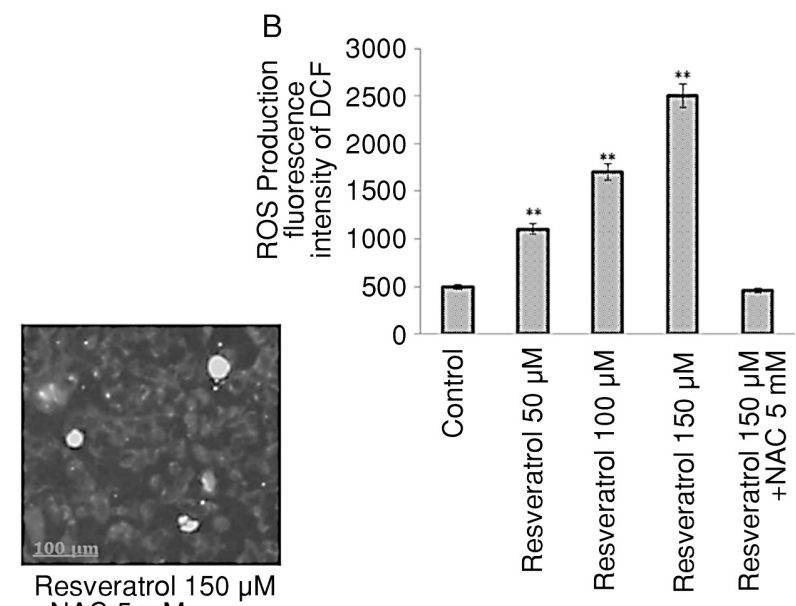

Figure 3. Resveratrol induces ROS production in pancreatic cancer cells. (A) Representative fluorescence microscopy images of DCFDA staining show Mia-PaCa cells that were exposed to varying concentrations of resveratrol along with untreated control, and negative control NAC, Scale bar: $100 \mu \mathrm{m}$; 20x. (B) The fluorescence intensity of resveratrol and NAC treated DCF cells was quantified by a microplate reader, from 3 independent experiments. Data represent the mean value of \pm SE of 3 independent experiments, $* * p<0.01$. 
A
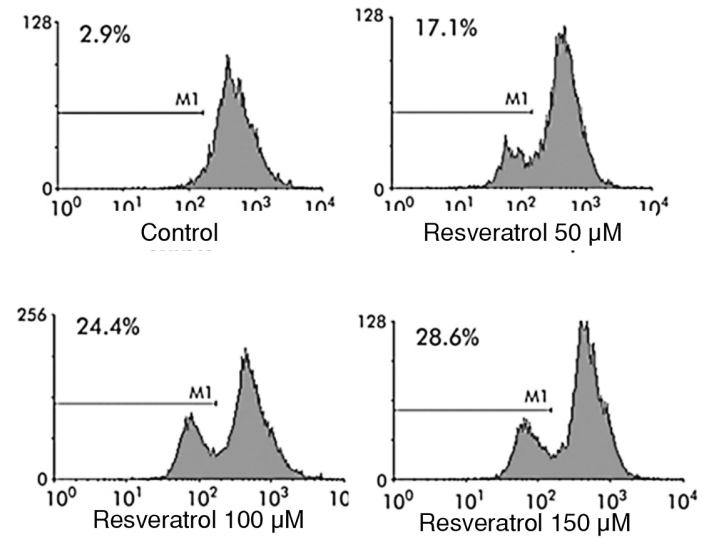

B

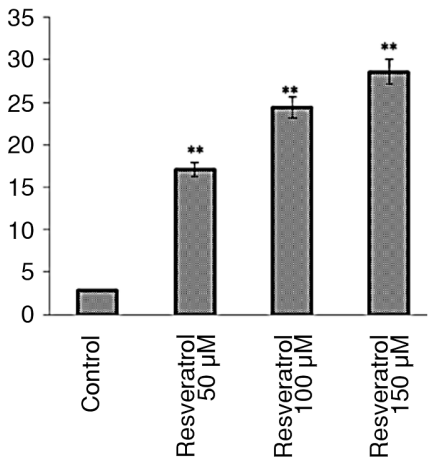

C

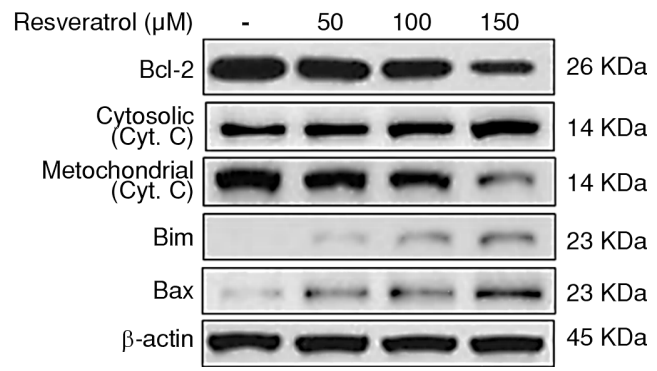

Figure 4. Measurement of mitochondrial membrane potential (MMP) pancreatic cells were treated with varying concentrations of resveratrol. (A) Representative flow cytometry images of pancreatic cells (Mia-PaCa) were exposed to varying doses $(50,100$, and $150 \mu \mathrm{M})$ of resveratrol along with untreated control for $24 \mathrm{~h}$, followed by staining with JC-1 staining to evaluate the depolarization of mitochondrial membrane potential. (B) Bar diagram showing the percentage of the cell which undergoes depolarization of mitochondrial membrane potential after PANC-1 cells were exposed to varying concentrations of resveratrol. (C) Immunoblotting analysis showing of significant increase of mitochondrial proteins cytochrome $\mathrm{C}$, Bim, and $\mathrm{Bax}$ in cytosolic fractionation with a concomitant decrease in the expression of anti-apoptotic protein Bcl-2 in Mia-PaCa cell lysates when exposed to indicated concentrations of resveratrol for $24 \mathrm{~h}$ along with untreated control. The data represent the mean value $\pm \mathrm{SE}$ of three independent experiments $* * \mathrm{p}<0.01$.

pancreatic cancer cells which was well supported by changes in cell and nuclear morphology in a dosedependent manner. Mechanistically, resveratrol promotes oxidative stress-mediated activation of the cell death mechanism by activates pro-apoptotic proteins caspase- 3 and PARP1. Further, the analysis revealed that resveratrol-mediated cell death in pancreatic cancer is due to mitochondrial-mediated apoptosis as evidenced by the loss of mitochondrial membrane potential and secretion of one of the hallmark and resident protein of mitochondria cytochrome $\mathrm{C}$ in the cytosolic fraction of cell lysate by immunoblotting. Additionally, we found significant ROS production induced in pancreatic cells after exposure to varying doses of resveratrol also supports the induction of mitochondrial-dependent apoptosis in pancreatic cells exposed to resveratrol treatment.

Owing to exhibit numerous pharmacological properties, resveratrol from the last two decades has gained tremendous attention as a potential antitumor agent against a wide variety of cancers with less deleterious effects on normal cells $(33,34)$. Here we studied the effect of resveratrol on cell viability of pancreatic cancer cells. We observe a significant reduction in cell viability of pancreatic cancer upon treatment with resveratrol with IC50 values in a range from $78.9 \mu \mathrm{M}$ to $90.34 \mu \mathrm{M}$ against a panel of pancreatic cancer cells. Natural compounds derived from plant source induces cytotoxic effect to tumor cells by either activates apoptosis mode of cell death or autophagic mode of cell death $(35,36)$. However, the most common mode of cell killing is programmed cell type I (apoptosis) (37). The striking features of apoptosis cell death are a disruption in membrane asymmetry with membrane blebbing, reduction in cell size followed by nuclear fragmentation and DNA fragmentation (38). Data from various laboratories revealed that resveratrol exhibits antiproliferative effects by modulating proapoptotic and antiapoptotic family proteins in favor of cell death. Miki et. al. demonstrated that resveratrol activates apoptosis in colorectal cancer cells by increasing the cleavage activity of caspase- 8 and caspase- 3 proteins which plays a key role in apoptosis cell death (39). A similar study in hepatocellular carci- 
noma cells revealed that resveratrol augments activation of caspase- 3 mediated apoptosis thus kills hepatocellular carcinoma cells (40). Consistent with the previous studies, our flow cytometry results revealed that a significant apoptotic cell population was quantified after pancreatic cells were exposed to a higher concentration of resveratrol. When confirmed the proteins involved in cell death of pancreatic cancer cells, our immunoblotting results revealed that the prominent cleavage fragments of proapoptotic proteins caspase-3 and PARP1 were observed on immunoblot lanes where pancreatic cells were exposed to higher doses of resveratrol.

Oxidative stress is one of the important prerequisites in tumorigenesis and drives tumor cells towards invasiveness in various cancers (41). ROS is one of the important components of oxidative stress and plays a crucial role in the development of tumorigenesis in a wide variety of cancers (42). A recent study demonstrates that resveratrol treatment induces ROS production in colorectal cancer cells and simultaneously induces autophagy and apoptosis (39). A similar study demonstrates that resveratrol exhibits an antiproliferative effect against lung cancer cells and simultaneously upregulates Nox5 expression and ROS production (43). Consistent with these findings our results showed that resveratrol induces ROS production when Mia-PaCa cells were exposed to varying doses of resveratrol. This augmentation of ROS generates oxidative stress in pancreatic cancer cells and leads to the activation of mitochondrial-mediated apoptosis mechanism of cell death. This was evidenced by the loss of mitochondrial membrane potential and secretion of mitochondrial resident protein cytochrome $\mathrm{C}$ in cytosolic fraction after pancreatic cells were exposed to varying doses of resveratrol. However, pancreatic cells exposed to a high dose of resveratrol in the presence of NAC inhibits ROS production profoundly. This indicates that NAC prevents oxidative stress-mediated activation of mitochondrial-dependent apoptosis by resveratrol in pancreatic cancer cells.

Numerous studies suggest that resveratrol exhibits chemopreventive activity due to its profound proapoptotic activity. Resveratrol selectively activates apoptotic mechanisms in human leukemia and mouse cells; however, hematopoietic progenitor cells are resistant to the apoptosis mechanism induced by resveratrol (44). Resveratrol treatment in breast cancer cells induces Fas/FasL-mediated apoptosis mechanism, however, in colorectal cancer cells resveratrol has been observed to induce mitochondrial-dependent apoptosis (45). In agreement with these studies, we observe a significant quantification of the population of pancreatic cancer cells that lose the mitochondrial membrane potential thereby undergoes mitochondrial-mediated apoptosis. Additionally, immunoblotting results reveal that resveratrol augments the secretion of cytochrome $\mathrm{C}$ in cytosolic fraction with a sharp decrease and increase in expression of anti-apoptotic protein $\mathrm{Bcl}$ 2 and proapoptotic protein Bax, respectively. Collectively, these results suggest that resveratrolmediates ROS production, which in turn augments mitochondrial-dependent apoptosis in pancreatic cancer.

\section{CONCLUSION}

In conclusion, the current study evaluates the anticancer potential of resveratrol against pancreatic cancer cells. Our data demonstrate that resveratrol sensitizes pancreatic cancer to cell death mechanisms by promotes oxidative stress-mediated ROS production and activation of the pro-apoptotic caspase cascade. Further, our study reveals that resveratrol induces mitochondrial-mediated apoptosis in pancreatic cancer cells which were evidenced by the loss of mitochondrial membrane potential and secretion of mitochondrial cytochrome C. Thus, our present findings offer a piece of compelling evidence for further evaluation of resveratrol in preclinical models to elucidate the efficacy, toxicity, and underlying mechanisms of cell death. These investigations will help to develop resveratrol as a promising chemotherapeutic agent and to address its utility as a potential future anticancer therapeutic agent against pancreatic cancer and associated malignancies.

\section{Acknowledgments}

The authors would like to thank the Department of Hepatobiliary Surgery, General Hospital of Ningxia Medical University, Yinchuan, Ningxia, China, 750004. Department of Colorectal Surgery, General Hospital of Ningxia Medical University, Yinchuan, Ningxia, China, 750004 for grant and support.

\section{Conflict of interest}

The authors declare no conflict of interest.

\section{REFERENCES}

1. Rahib L., Smith B.D., Aizenberg R., Rosenzweig A.B., Fleshman J.M., Matrisian L.M.: Cancer Res. 74, 2913 (2014). 
2. Freelove R., Walling A.: Am. Fam. Physician 73, 485 (2006).

3. Kamisawa T., Wood L.D., Itoi T., Takaori K.: Lancet 388, 73 (2016).

4. Garcea G., Dennison A.R., Pattenden C.J., Neal C.P., Sutton C.D., Berry D.P.: JOP 9, 99 (2008).

5. Midha S., Chawla S., Garg P.K.: Cancer Lett. 381, 269 (2016).

6. Bailey P., Chang D.K., Nones K., Johns A.L., Patch A.-M., et al.: Nature 531, 47 (2016).

7. Macgregor-Das A.M., Iacobuzio-Donahue C.A.: J. Surg. Oncol. 107, 8 (2013).

8. Seoane J., Gomis R.R.: Cold Spring Harb. Perspect. Biol. 9, a022277 (2017).

9. Singh P., Srinivasan R., Wig J.D.: J. Pancreas 40, 644 (2011).

10. Rossi M.L., Rehman A.A., Gondi C.S.: World J. Gastroenterol. 20, 11142 (2014).

11. Lee J.-L., Kim S.C., Kim J.-H., Lee S.S., Kim T.-W., et al.: Surgery 152, 851 (2012).

12. Erkan M., Hausmann S., Michalski C.W., Fingerle A.A., Dobritz M., et al.: Nat. Rev. Gastroenterol. Hepatol. 9, 454 (2012).

13. Feitelson M.A., Arzumanyan A., Kulathinal R.J., Blain S.W., Holcombe R.F., et al.: Semin. Cancer Biol. 35 (Suppl) S25 (2015).

14. Millimouno F.M., Dong J., Yang L., Li J., Li X.: Cancer Prev. Res. 7, 1081 (2014).

15. Borriello A., Cucciolla V., Della Ragione F., Galletti P.: Nutr. Metab. Cardiovasc. Dis. 20, 618 (2010).

16. Plumed-Ferrer C., Väkeväinen K., Komulainen H., Rautiainen M., Smeds A., et al.: Int. J. Food Microbiol. 164, 99 (2013).

17. Frémont L.: Biological effects of resveratrol. Life Sci. 66, 663 (2000).

18. Yanez M., Blanchette J., Jabbarzadeh E.: Curr. Pharm. Des. 23, 6347 (2017).

19. Pangeni R., Sahni J.K., Ali J., Sharma S., Baboota S.: Expert Opin. Drug Deliv. 11, 1285 (2014).

20. Athar M., Back J.H., Tang X., Kim K.H., Kopelovich L., et al.: Toxicol. Appl. Pharmacol. 224, 274 (2007).

21. Alkhalaf M., El-Mowafy A., Renno W., Rachid O., Ali A., Al-Attyiah R.: Arch. Med. Res 39, 162 (2008).

22. Delmas D., Rebe C., Micheau O., Athias A., Gambert P., et al.: Oncogene 23, 8979 (2004).

23. Fulda S., Debatin K.-M.: Eur. J. Cancer 41, 786 (2005).
24. Ilic M., Ilic I.: World J. Gastroenterol.22, 9694 (2016)

25. Westphalen C.B., Kruger S., Haas M., Heinemann V., Boeck S.: Expert Opin. Drug Saf. 15, 947 (2016).

26. Kleeff J., Korc M., Apte M., La Vecchia C., Johnson C.D.: et al.: Nat. Rev. Disease Primers 2, 1 2016).

27. Grant T.J., Hua K., Singh A.: Prog. Mol. Biol. Trans. Sci. 144, 241 (2016).

28. Antoniou G., Koutsounas I., Kountourakis P., Pontas C., De Mello R.A.: Pancreatic Cancer, International Manual of Oncology Practice. pp. 343-392, Springer 2015.

29. Bishayee A.: Cancer Prev. Res 2, 409 (2009).

30. Athar M., Back J.H., Kopelovich L., Bickers D.R., Kim A.L.: Arch. Biochem. Biophys. 486, 95 (2009).

31. Scarlatti F., Maffei R., Beau I., Codogno P., Ghidoni R.: Cell Death Differ. 15, 1318 (2008).

32. Benitez D.A., Pozo-Guisado E., AlvarezBarrientos A., Fernandez-Salguero P.M., Castellón E.A.: J. Androl. 28, 282 (2007).

33. Panda A.K., Chakraborty D., Sarkar I., Khan T., Sa G.: J. Exp. Pharmacol. 9, 31 (2017).

34. Sinha D., Sarkar N., Biswas J., Bishayee A.: Semin. Cancer Biol. 40, 209 (2016).

35. Fulda S., Kögel D.: Oncogene 34, 5105 (2015).

36. Gali-Muhtasib H., Hmadi R., Kareh M., Tohme R., Darwiche N.: Apoptosis 20, 1531 (2015).

37. Maghsoudi N., Zaketi Z., Lockshin R.: Exp. Oncol. 34, 146 (2012).

38. Wlodkowic D., Telford W., Skommer J., Darzynkiewicz Z.: Methods Cell Biol. 103, 55 (2011)

39. Miki H., Uehara N., Kimura A., Sasaki T., Yuri T., et al.: Int. J. Oncol. 40, 1020 (2012).

40. Lin C.Y., Chang T.W., Hsieh W.H., Hung M.C., Lin I.H, et al.: Cell Death Discov. 2, 16065 (2016).

41. Chikara S., Nagaprashantha L.D., Singhal J., Horne D., Awasthi S., Singhal S.S.: Cancer Lett. 413, 122 (2018).

42. Waris G., Ahsan H.: J. Carcinog. 5, 14 (2006).

43. Yousef M., Vlachogiannis I.A., Tsiani E.: Nutrients 9, 1231 (2017).

44. Dörrie J., Gerauer H., Wachter Y., Zunino S.J.: Cancer Res. 61, 4731 (2001).

45. Su J.L., Lin M.T., Hong C.C., Chang C.C., Shiah S.G., et al.: Carcinogenesis 26, 1 (2005).

(C) 2020 by Polish Pharmaceutical Society. This is an access article under the CC BY NC license (http://creativecommons.org/licenses/by-nc/4.0/). 